\title{
Central Brain Herniation Secondary to Influenza-Related Encephalopathy in a Pontocerebellar Hypoplasia Child: A Case Report and Review
}

\author{
Weiqiang Xiao', Peiqing Li², Yuzhi Yao3 ${ }^{3}$, Sida Yang", \\ Suyun Li², Shuyao Ning5, Hongsheng Liu ${ }^{*}$
}

\footnotetext{
${ }^{1}$ Department of Pediatric Radiology, Guangzhou Women and Children's Medical Center, Guangzhou Medical University, Guangzhou, China

${ }^{2}$ Department of Pediatric Emergency, Guangzhou Women and Children’s Medical Center, Guangzhou Medical University, Guangzhou, China

${ }^{3}$ Department of Surgery, Guangzhou Women and Children's Medical Center, Guangzhou Medical University, Guangzhou, China ${ }^{4}$ Department of Pediatric Neurology, Guangzhou Women and Children's Medical Center, Guangzhou Medical University, Guangzhou, China

${ }^{5}$ Department of Neuroelectrophysiology, Guangzhou Women and Children's Medical Center, Guangzhou Medical University, Guangzhou, China

Email: *liuhs72@163.com
}

How to cite this paper: Xiao, W.Q., Li, P.Q., Yao, Y.Z., Yang, S.D., Li, S.Y., Ning, S.Y. and Liu, H.S. (2020) Central Brain Herniation Secondary to Influenza-Related Encephalopathy in a Pontocerebellar Hypoplasia Child: A Case Report and Review. Journal of Behavioral and Brain Science, 10, 257-264.

https://doi.org/10.4236/jbbs.2020.106016

Received: May 11, 2020

Accepted: June 14, 2020

Published: June 17, 2020

Copyright $\odot 2020$ by author(s) and Scientific Research Publishing Inc. This work is licensed under the Creative Commons Attribution International License (CC BY 4.0).

http://creativecommons.org/licenses/by/4.0/ (c) (i) Open Access

\begin{abstract}
Influenza-associated encephalopathy (IAE) can perform as varying patterns of neuroimaging. Central brain herniation $(\mathrm{CBH})$ secondary to IAE is rare; it may be a bad prognosis. Here, we presented a 4-year-old girl with influenza who had a pontocerebellar hypoplasia $(\mathrm{PCH})$ history; she performed the second Magnetic Resonance Imaging (MRI) on Day 6 from onset, showed the diffuse edema and the occurrence of central herniation; the medulla was "Z-like" folded and the basal cisterns were obliterated completely. Finally she was declared dead. The imbalance between supratentorial and infratentorial pressure can lead to the occurrence of $\mathrm{CBH}$. Severe edema relates to IAE and unstable structure of the posterior fossa might be the main reason for the herniation. MRI is helpful in early diagnosis. Early treatment of cerebral edema in patients with congenital abnormalities of the posterior fossa is vital for their management.
\end{abstract}

\section{Keywords}

Pontocerebellar Hypoplasia, Central Brain Herniation, Influenza-Related Encephalopathy, Magnetic Resonance Imaging 


\section{Introduction}

Influenza in children is a major cause of morbidity and mortality worldwide. Annual epidemics in adults and children are associated with about 290,000 650,000 deaths. Neurological complications associated with influenza include febrile seizures, influenza-associated encephalitis/encephalopathy (IAE), etc. [1] [2]. IAE can perform as varying patterns of neuroimaging. Some patients manifested as diffuse edema of the cerebral cortex and the involvement of basal ganglia. Though only rare cases are severe enough to cause central brain herniation (CBH) [3], the patients with cystic abnormalities or shunting history of posterior fossa might at higher risk of $\mathrm{CBH}$. $\mathrm{CBH}$ caused by IAE is rare, but it can lead to high mortality. Central brain herniation $(\mathrm{CBH})$, also known as bilateral transtentorial herniation, may occur as a complication of bilateral supratentorial intracranial expansive processes (often acute traumatic or vascular lesions) [4]. Compression of the respiratory and cardiac centers of the reticular substance may lead to irreversible coma or death. Central herniation is the end result of downward displacement of the cerebral hemispheres and the basal nuclei compressing and displacing the diencephalon and the midbrain rostrocaudally through the tentorial notch. There may be herniation also of both temporal unci and parahippocampal gyri as well as the lingual gyrus and isthmus of the gyrus fornicatus into the tentorial hiatus [4] [5] [6]. The brain stem is compressed transversely and will appear elongated on its anteroposterior axis on axial imaging. The basal cisterns may be obliterated completely, especially the perimesencephalic (interpeduncular, quadrigeminal, ambient, and crural) and suprasellar cisterns [4] [6]. There may be downward shifting of the basilar artery and pineal gland [7].

\section{Case Report}

We present a 4-year-old girl who got the type A influenza with high fever onset, rapidly developing frequent convulsions. On the 2nd day from onset, she had acute disorder of consciousness (ADOC), which led her emergency admission. She completed her first brain MRI on the same day and was diagnosed as pontocerebellar hypoplasia (PCH) [8], no MRI sign of infection was noted (Figure 1(a), Figure 1(c), Figure 1(e)). She had backward neurodevelopment history; she could not stand alone until being over 1 year old and walk alone until being over 2 years old, but there was no further exam until this admission. Table 1 shows sociodemographic and clinical characteristics in this case. After admission, the patient was given Oseltamivir and intravenous immunoglobulin treatment in accordance with the "Protocol for diagnosis and treatment of influenza (2018 revised version)" [9] issued by the General Office of the National Health and Health Commission. And she was given mannitol and hypertonic saline for dehydration when she was transferred into the pediatric intensive care unit, advanced life support in time when her condition changed.

On the $5^{\text {th }}$ day from onset, she suddenly had irregular breathing rhythm, 
Table 1. Sociodemographic and clinical characteristics.

\begin{tabular}{|c|c|}
\hline Characteristics & Data \\
\hline Nationality & China \\
\hline Age (years) & 4 \\
\hline Gender & Female \\
\hline Weight (kg) & 15 \\
\hline Mother's Gravidity and parity history & G4P4 \\
\hline Delivery mode & Caesarean \\
\hline Birth (full term/premature) & full term \\
\hline Birth weight $(\mathrm{kg})$ & 3 \\
\hline \multicolumn{2}{|l|}{ Clinical characteristics } \\
\hline Consciousness & Acute Disorder of Consciousness \\
\hline Glasgow Coma Scale & 3 \\
\hline Pupillary & anisocoria \\
\hline Pupillary light reflex & absent \\
\hline Breathing Rhythm & irregular \\
\hline Heart Rate (beats per minute) & 216 \\
\hline Infarction & $\begin{array}{l}\text { territories supplied by the posterior cerebral, thalamoperforate, } \\
\left.\text { and superior cerebellar arteries (diagnosed by her } 2^{\text {nd }} M R I\right)\end{array}$ \\
\hline Etiology of pharyngeal swab PCR & Influenza A \\
\hline \multicolumn{2}{|l|}{ Routine blood test } \\
\hline Hemoglobin (g/L) & 100 \\
\hline White blood cells $\left(\times 10^{9} / \mathrm{L}\right)$ & 9.6 \\
\hline Neutrophils $\left(\times 10^{9} / \mathrm{L}\right)$ & 7.1 \\
\hline Lymphocytes $\left(\times 10^{9} / \mathrm{L}\right)$ & 1.63 \\
\hline Monocytes $\left(\times 10^{9} / \mathrm{L}\right)$ & 0.86 \\
\hline Platelets $\left(\times 10^{9} / \mathrm{L}\right)$ & 288 \\
\hline \multicolumn{2}{|l|}{ CSF test after admission } \\
\hline White blood cells $\left(\times 10^{9} / \mathrm{L}\right)$ & 1 \\
\hline C-reactive protein $(\mathrm{mg} / \mathrm{mL})$ & 0.05 \\
\hline Chloride $(\mathrm{mEq} / \mathrm{L})$ & 126 \\
\hline Glucose $(\mathrm{mmol} / \mathrm{L})$ & 4.05 \\
\hline $\mathrm{MP}(\mathrm{g} / \mathrm{L})$ & 0.12 \\
\hline Lactate dehydrogenase (U/L) & 221 \\
\hline CSF pressure $\left(\mathrm{mmH}_{2} \mathrm{O}\right)$ & 250 \\
\hline \multicolumn{2}{|l|}{ Biochemistry } \\
\hline $\operatorname{ALT}(\mathrm{U} / \mathrm{L})$ & 122 \\
\hline $\mathrm{AST}(\mathrm{U} / \mathrm{L})$ & 363 \\
\hline
\end{tabular}




\section{Continued}

\begin{tabular}{cc}
\hline ALT/AST & 0.34 \\
Lactate dehydrogenase (U/L) & 1129 \\
Creatinine kinase (U/L) & 4083 \\
Creatinine kinase MB (U/L) & 169 \\
Ammonia $(\mu \mathrm{M} / \mathrm{L})$ & 27.7 \\
\hline
\end{tabular}

\section{Day2}
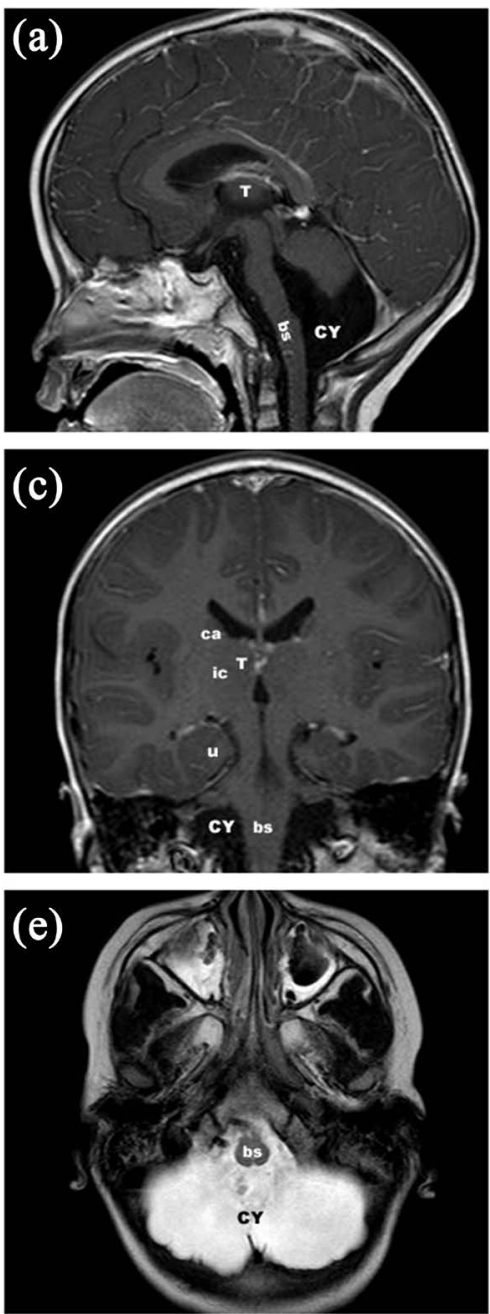

\section{Day6}
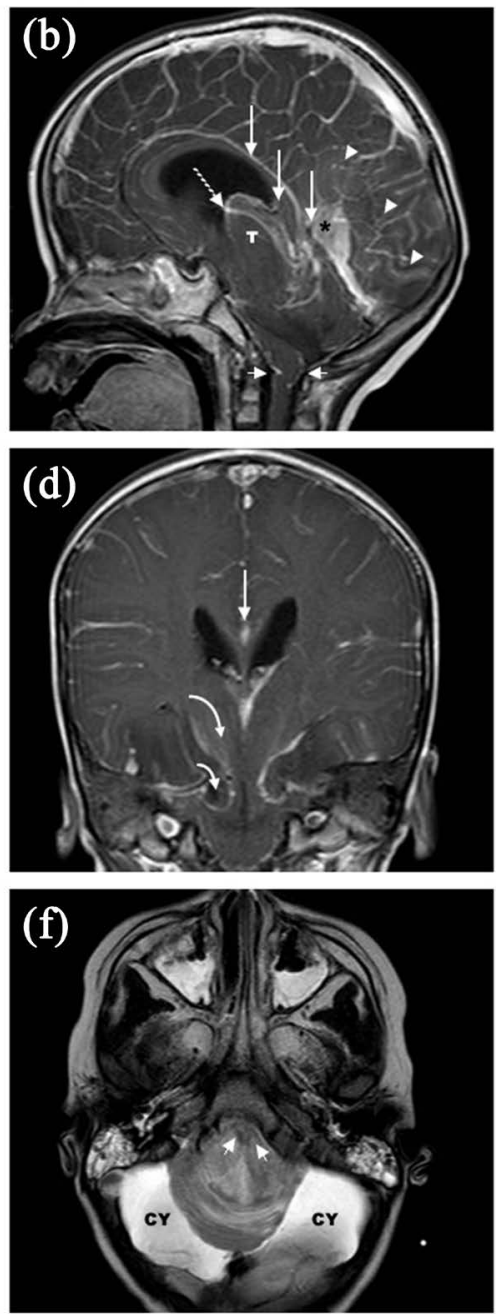

Note: T: thalami; bs: brainstem; CY: cyst; ca: nucleus caudatus; ic: inner capsule; u: uncinate gyrus.

Figure 1. Comparison before and after the occurrence of CBH secondary by IAE. (a) (c) (e) MRI on day 2, the hypoplastic of inferior vermis and pons, and the cystic space of the posterior fossa was seen, the fourth ventricle was large but less dilated than the classic Dandy-Walker malformation. No MRI sign of infection was noted. (b) (d) (f) MRI on day 6 , Bilateral thalami were downward shifted significantly (dashed arrow), the inner capsule, temporal unci and parahippocampal gyri were herniated (curved arrow) and the medulla was "Z-like" folded (short arrow). The basal cisterns were obliterated completely, the volume of the posterior fossa cystic space (CY) decreased and the bilateral lateral ventricles were expanded. The inferior sagittal sinus, internal cerebral vein were pushed downward (long arrow), the Galen vein was mildly expanded $\left(^{*}\right)$. 
acute pulmonary edema, anisocoria and disappearance of pupillary light reflection, and then deep coma. She performed her second brain MRI on the next day (Figure 1(b), Figure 1(d), Figure 1(f)), showing that the diffuse edema of the cerebral cortex and basal ganglia, meanwhile the occurrence of central herniation. The diencephalon, midbrain, temporal unci and parahippocampal gyri were significantly displaced, and the medulla was "Z-like" folded and the basal cisterns were obliterated completely. Furthermore, infractions of right medial occipital lobe were seen in the territory of posterior cerebral artery (Figure 2). The volume of the posterior fossa cystic space decreased from $776.86 \mathrm{~mm}^{3}$ to $197.76 \mathrm{~mm}^{3}$. Her biochemical and routine indicators of CSF were normal but CSF-pressure was as high as $250 \mathrm{mmH}_{2} \mathrm{O}$, which could be clinically diagnosed as central herniation secondary to influenza-associated encephalopathy (IAE) with intracranial hypertension. Despite treatment with dehydration, fluid restriction, antiviral, anti-infective and supportive therapy were given, the patient developed progressive exacerbations and was finally declared brain dead by twice brain function evaluations by electroencephalogram and transcranial doppler.

\section{Discussion}

The imbalance between supratentorial and infratentorial pressure can lead to the occurrence of $\mathrm{CBH}$. The common causes of pressure increasing include: cerebral edema secondary to infection or trauma, stroke, brain hemorrhage, brain tumor, other reasons include abscess, hydrocephalus, shunting or other brain surgery. Table 2 summarizes different possible etiology to explain this case. IAE can perform as varying patterns of neuroimaging. According to their different radiographic features, Kimura S [10] divided them into five categories: normal (category 1); diffuse involvement of the cerebral cortex (category 2); diffuse brain edema (category 3); symmetrical involvement of the thalamus (category 4); and postinfectious focal encephalitis (category 5). Most severe cases of them are
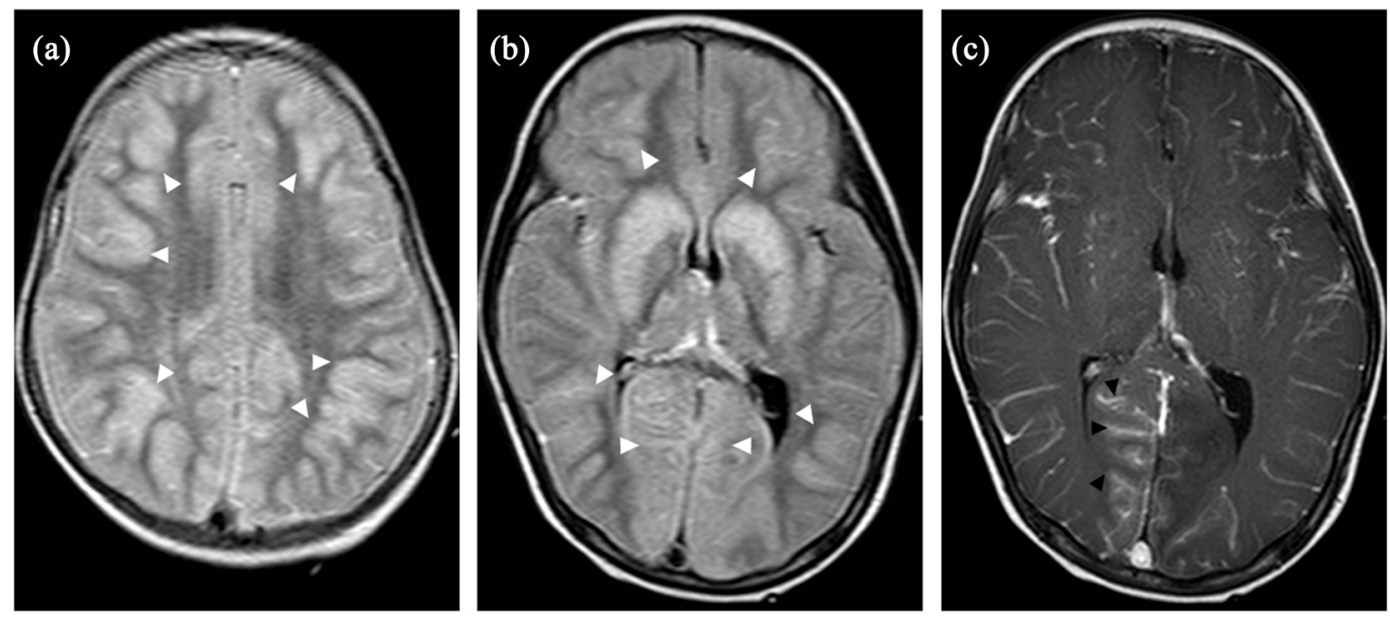

Figure 2. MRI on day 6, the gyrus of frontal and parietal and occipital lobes (white arrowhead) and the basal ganglia were involved by influenza infection. Infractions of the right medial occipital lobe were enhanced obviously (black arrowhead). 
Table 2. Possible etiology for central brain herniation in this case.

\begin{tabular}{|c|c|}
\hline $\begin{array}{l}\text { Supratentorial volumn } \\
\text { and pressure increasing } \\
\text { factors }\end{array}$ & $\begin{array}{l}\text { 1) Swelling gyrus and basal ganglia caused by IAE } \\
\text { 2) Intermittent chronic occlusion of the aqueduct resulting in gradual } \\
\text { increase in ventricular pressure } \\
\text { 3) Edema caused by vascular compression of the central herniation }\end{array}$ \\
\hline $\begin{array}{l}\text { Infratentorial volumn and } \\
\text { pressure decreasing factors }\end{array}$ & $\begin{array}{l}\text { Decreased volumn of the posterior fossa cystic space of } \mathrm{PCH} \text { created the } \\
\text { space and negative pressure gradient }\end{array}$ \\
\hline Other & Idiopathic \\
\hline
\end{tabular}

category 4, some develop to acute necrotizing encephalitis of childhood [11] (ANE), category 2 and category 3 are relatively few, CBH in these cases are rare. In our patient with $\mathrm{PCH}$, IAE rapidly developed and manifested as diffuse edema of the cerebral cortex and basal ganglia which can be classified into category 2 and 3, it created a positive pressure gradient over the tentorium and increased the risk of cerebral herniation. Severe intracranial hypertension relates to IAE has been reported [12]. If the elevation of intracranial pressure caused by expansion of intracranial volume is beyond its ability to compensate, the cerebral herniation occurs. However, only rarely is the edema severe enough to cause $\mathrm{CBH}$ [1]. Such predisposition may be aggravated by the compression of the aqueduct and vascular from downward transincisural herniation, even became a vicious cycle. The posterior fossa cystic space and the hypoplastic of the inferior vermis and pons in our case, may provide the space of downward transincisural herniation. What caused the posterior fossa cystic space to shrink is uncertain, maybe the elevation of the supratentorial pressure or the unstable structures of the space itself.

The clinical manifestations of central herniation depend on the degree of compression or displacement of anatomic structures. First usually the change in alertness or behavior secondary to diencephalic dysfunction followed by respiratory, ocular, and motor signs. As the disease progresses, uncal and parahippocampal herniation may result in compression of the third nerve and tectum, leading to unilateral or bilateral third-nerve palsy, failure of upward gaze, and loss of the pupillary light reflex. When the herniation is of sufficient severity, obstruction of the aqueduct may occur leading to increase intraventricular pressure and varying degrees of hydrocephalus and papilledema [2] [3]. Infarction in territories supplied by the posterior cerebral, thalamoperforate, and superior cerebellar arteries resulting from vascular compression at the tentorial notch have been described [13] [14]. We can see most of the signs and symptoms above in our case. Finally, the compression of the respiratory and cardiac centers of brain stem leads to irreversible coma or death.

Shrier [15] reported a case of central brain herniation secondary to the brain edema of juvenile diabetic ketoacidosis. Mandiwanza [16] reported a case of central brain herniation in shunted Dandy walker cyst. Naidich [17] reported 7 cases of chronic central brain herniation $(\mathrm{CCBH})$ in shunted Dandy walker malformation, two of them died. These cases above suggested that $\mathrm{CBH}$ will oc- 
cur by different reasons for the pressure changing over or under the tentorium, it may be a bad prognosis in patients with central herniation.

\section{Conclusion}

This PCH child having $\mathrm{CBH}$ secondary to IAE is uncommon. To our knowledge this is the first radiologic literature regarding CCBH secondary to IAE without treatment of congenital posterior fossa cystic space. In this case, we can learn that MRI is helpful for early diagnosis; timely imaging examinations can make us understand possible etiology of the herniation, speed and degree of the primary disease, evaluate the severity of cerebral hernia with patient's neurologic state, and provide more information for treatment and prognosis evaluation accurately. The lessons we can learn from this rare case are, some IAE cases might develop rapidly; once neurological symptoms were found in influenza children with unstable structural abnormality or shunting history of posterior fossa, more active dynamic observation of their clinical performances and positive assessment monitoring should be given, timely imaging examinations such as bedside ultrasound and MRI are needed for discovering of the happening of early brain edema. Early diagnosis and treatment of diffuse brain edema is essential for their survival.

\section{Acknowledgements}

This work was supported by: The Technology Planning Project of Guangzhou City (No. 201804010142).

\section{Conflicts of Interest}

The authors declare no conflicts of interest regarding the publication of this paper.

\section{References}

[1] Yildizdaş, D., Kendirli, T. and Arslanköylü, A.E. (2011) Neurological Complications of Pandemic Influenza (H1N1) in Children. European Journal of Pediatrics, 170, 779-788. https://doi.org/10.1007/s00431-010-1352-y

[2] Maricich, S.M., Neul, J.L. and Lotze, T.E. (2004) Neurologic Complications Associated with Influenza A in Children during the 2003-2004 Influenza Season in Houston, Texas. Pediatrics, 114, e626-e633. https://doi.org/10.1542/peds.2004-0143

[3] Duck, S.C. and Wyatt, D.T. (1988) Factors Associated with Brain Herniation in the Treatment of Diabetic Ketoacidosis. The Journal of Pediatrics, 113, 10-14. https://doi.org/10.1016/S0022-3476(88)80521-3

[4] Nguyen, J.P., Djindjian, M., Brugieres, P., Badiane, S., Melon, E. and Poirier, J. (1989) Anatomy-Computerized Tomography Correlations in Transtentorial Brain Herniation. Journal of Neuroradiology, 16, 181-196.

[5] Laine, F.J., Shedden, A.I., Dunn, M.M. and Ghatak, N.R. (1995) Acquired Intracranial Herniations: MR Imaging Findings. American Journal of Roentgenology, 165, 967-973. https://doi.org/10.2214/ajr.165.4.7677003 
[6] Stovring, J. (1977) Descending Tentorial Herniation: Findings on Computed Tomography. Neuroradiology, 14, 101-105. https://doi.org/10.1007/BF00333050

[7] Hahn, F.J. and Gurney, J. (1985) CT Signs of Central Descending Transtentorial Herniation. American Journal of Neuroradiology, 6, 844-845.

[8] Bosemani, T., Orman, G., Boltshauser, E., Tekes, A., Huisman, T.A. and Poretti, A. (2015) Congenital Abnormalities of the Posterior Fossa. Radiographics, 35, 200-220. https://doi.org/10.1148/rg.351140038

[9] National Health Commission of the People's Republic of China (2019) Protocol for Diagnosis and Treatment of Influenza (2018 Revised Version). Zhonghua Linchuang Ganranbing Zazhi, 12, 1-5.

[10] Kimura, S., Ohtuki, N., Nezu, A., Tanaka, M. and Takeshita, S. (1998) Clinical and Radiological Variability of Influenza-Related Encephalopathy or Encephalitis. Acta Paediatrica Japonica: Overseas Edition, 40, 264-270. https://doi.org/10.1111/j.1442-200X.1998.tb01925.x

[11] Dadak, M., Pul, R., Lanfermann, H., et al. (2019) Varying Patterns of CNS Imaging in Influenza A Encephalopathy in Childhood. Clinical Neuroradiology. https://doi.org/10.1007/s00062-018-0756-3

[12] Citerio, G., Sala, F., Patruno, A., et al. (2010) Influenza A (H1N1) Encephalitis with Severe Intracranial Hypertension. Minerva Anestesiologica, 76, 459-462.

[13] Endo, M., Ichikawa, F., Miyasaka, Y., Yada, K. and Ohwada, T. (1991) Capsular and Thalamic Infarction Caused by Tentorial Herniation Subsequent to Head Trauma. Neuroradiology, 33, 296-299. https://doi.org/10.1007/BF00587809

[14] Eskandar, E.N., Weller, S.J. and Frim, D.M. (1997) Hydrocephalus Requiring Urgent External Ventricular Drainage in a Patient with Diabetic Ketoacidosis and Cerebral Edema: Case Report. Neurosurgery, 40, 836-838; discussion 838-839. https://doi.org/10.1097/00006123-199704000-00035

[15] Shrier, D.A., Shibata, D.K., Wang, H.Z., Numaguchi, Y. and Powers, J.M. (1999) Central Brain Herniation Secondary to Juvenile Diabetic Ketoacidosis. American Journal of Neuroradiology, 20, 1885-1888.

[16] Mandiwanza, T., Kaliaperumal, C. and Caird, J. (2013) Central Brain Herniation in Shunted Dandy Walker Cyst. Child s Nervous System, 29, 1035-1038. https://doi.org/10.1007/s00381-013-2066-Z

[17] Naidich, T.P., Radkowski, M.A., McLone, D.G. and Leestma, J. (1986) Chronic Cerebral Herniation in Shunted Dandy-Walker Malformation. Radiology, 158, 431-434. https://doi.org/10.1148/radiology.158.2.3941868 